Algebra and Logic, Vol. 43, No. 6, 2004

\title{
THE COMPUTABLE DIMENSION OF I-TREES OF INFINITE HEIGHT
}

\author{
N. T. Kogabaev, O. V. Kudinov, and R. Miller*
}

UDC $510.53+512.562$

Key words: computable tree with distinguished initial subtree, computable dimension, computably categorical model, branching model, effectively infinite computable dimension.

We study computable trees with distinguished initial subtree (briefly, I-trees). It is proved that all I-trees of infinite height are computably categorical, and moreover, they all have effectively infinite computable dimension.

In a finite language, a model $\mathfrak{A}$ is computable if its domain is a computable subset of $\omega$, and its basic operations and relations are all computable. In computable model theory, algorithmic properties of algebraic systems are treated up to computable isomorphism. The number of distinct (up to computable isomorphism) computable presentations of a model $\mathfrak{A}$ is called the computable dimension of $\mathfrak{A}$. If this dimension is 1 then we say that $\mathfrak{A}$ is computably categorical.

The computable categoricity of trees was studied in [1, 2]. In [1], it was proved that all computable trees of infinite height have computable dimension $\omega$. For computable trees of finite height, in [2], it was shown that their computable dimension may assume only the value 1 or $\omega$, and a complete characterization of computable categoricity was given.

In the present paper, we study the question about spectrum of possible computable dimensions of trees enriched by an initial subtree (briefly, $I$-trees). It is proved that the computable dimension of any computable $I$-tree of infinite height is $\omega$. Moreover, this dimension is effectively infinite, in the sense that, given any uniformly presented list of computable copies of the same $I$-tree, we can construct another computable copy of that tree, which is not computably isomorphic to any of the copies on the list. Notice that the results obtained can be naturally generalized to the case of several distinguished initial subtrees.

\section{THE NOTATION AND BASIC DEFINITIONS}

The notation and basic definitions on computable models are standard and can be found, for instance, in $[3,4]$. But our definitions on trees demand attention here.

A tree with distinguished initial subtree is a triple $(T, \prec, I)$ satisfying the following two conditions:

(1) A relation $\prec$ is a strict partial order on $T$ such that for every $x \in T$, the set of all predecessors of $x$ in $T$ is well ordered by $\prec$, and $T$ contains a least element $r$ under $\prec(r$ is called a root $)$.

(2) A subset $I \subseteq T$ is an initial subtree of $T$, that is,

$$
\forall x \forall y((x \in T \& y \in I \& x \prec y) \rightarrow x \in I) .
$$

*Supported by RFBR grant No. 02-01-00593, by the Council for Grants (under RF President) and State Aid of Fundamental Science Schools, project NSh-2112.2003.1, and by NSF grant No. 9983660.

Translated from Algebra i Logika, Vol. 43, No. 6, pp. 702-729, November-December, 2004. Original article submitted February 19, 2003.

0002-5232/04/4306-0393 (c) 2004 Springer Science+Business Media, Inc. 
Throughout, the trees with distinguished initial subtree are briefly called $I$-trees and are denoted by $(T, I)$. Hence the tree with distinguished initial subtree $(T, \prec, I)$ is computable if $T$ is a computable set, and both $\prec$ and $I$ are computable relations. If an $I$-tree has infinite height then without loss of generality we may assume the universe of $T$ to be $\omega$, pulling back via 1-1 computable function if necessary to make this so.

If two nodes $x$ and $y$ in $T$ are incomparable under $\prec$, then we write $x \perp y$. For each node $x \in T$, we define the level of $x$ in $T$ to be the order type of the set of predecessors of $x$ in $T$, and we denote it by $\operatorname{level}_{T}(x)$. Thus the level of the root is 0 , its immediate successors under $\prec$ are at level 1 , and so on. The height of $T$ is defined as follows:

$$
\operatorname{ht}(T)=\sup _{x \in T}\left(\operatorname{level}_{T}(x)+1\right) .
$$

If $x$ is a node in $T$, then by $T[x]$ we denote the subtree

$$
T[x]=\{y \in T \mid x \preccurlyeq y\} .
$$

The partial order on $T[x]$ is the restriction to $T[x]$ of the partial order $\prec$ on $T$. Therefore $T[x]$ is a subtree of $T$ with root $x$. The height of $T$ above $x$ is defined as follows:

$$
\operatorname{ht}_{x}(T)=\operatorname{ht}(T[x]) .
$$

A path through a tree $T$ is a maximal linearly ordered subset of $T$. A node is extendible if it lies on an infinite path through $T$, and non-extendible otherwise. The extendible nodes of $T$ (if any) form a subtree of $T$, which we denote by $T_{\text {ext }}$.

In this paper an embedding of one partial ordering $\left(T_{1}, \prec_{1}, I_{1}\right)$ with extra relation $I_{1} \subseteq T_{1}$ into another partial ordering $\left(T_{2}, \prec_{2}, I_{2}\right)$ with extra relation $I_{2} \subseteq T_{2}$ will be a one-to-one mapping $f: T_{1} \rightarrow T_{2}$ which respects the partial orders and the extra relations:

$$
x \prec_{1} y \Leftrightarrow f(x) \prec_{2} f(y), \quad x \in I_{1} \Leftrightarrow f(x) \in I_{2} .
$$

Moreover, if, in the previous definition, $\left(T_{1}, \prec_{1}, I_{1}\right)$ and $\left(T_{2}, \prec_{2}, I_{2}\right)$ are submodels of some partial ordering $(T, \prec, I)$ with extra relation $I \subseteq T$, that is, $\prec_{k}=\prec \cap T_{k}^{2}$ and $I_{k}=T_{k} \cap I$ for $k \in\{1,2\}$, then we say that $f: T_{1} \rightarrow T_{2}$ is an $I$-embedding.

For elements $x$ and $y$ of a tree, $x \wedge y$ denotes the infimum (if it exists) of $x$ and $y$. In some papers, all embeddings of trees are required to respect the infimum function. The latter requirement is stronger: any one-to-one map respecting $\wedge$ respects $\prec$, but not conversely. Kruskal's theorem, which we will use in Sec. 2, proves the existence of the stronger type of embeddings.

To prove that the computable dimension of some $I$-tree is effectively infinite, we use the branching models method, brought in sight in [5]. The method allows us to obtain necessary conditions for models in many classes of algebraic systems to be computably categorical (without using straight priority constructions). A number of generalizations and modifications of this method have been worked up to date (see [3]). We will need the following two versions of the theorem on branching models, the first of which was proven in [6].

Let $L$ be a finite predicate language, and let $\mathfrak{A}$ and $\mathfrak{B}$ be models for $L$. We write $\mathfrak{A} \leq \mathfrak{B}$ if $\mathfrak{A}$ is a submodel of $\mathfrak{B}$. By writing $\mathfrak{A} \equiv_{1} \mathfrak{B}$ we mean that the same $\exists$-sentences in $L$ are true in $\mathfrak{A}$ and in $\mathfrak{B}$.

First we formulate a definition of branching, necessary for the first version of the theorem. Let $\mathfrak{A}$ be an infinite computable model for a language $L$, and let $\left\{\mathfrak{A}_{p}\right\}_{p \in \omega}$ be a computable sequence of finite models for $L$ such that $\mathfrak{A}_{p} \leq \mathfrak{A}_{p+1} \leq \mathfrak{A}$ for each $p$, and $\mathfrak{A}=\bigcup_{p} \mathfrak{A}_{p}$. Further, let $\left\{\bar{c}_{p}\right\}_{p \in \omega}$ be a computable 
sequence of finite (possibly empty) tuples from $\mathfrak{A}$ with $\bar{c}_{p} \in \mathfrak{A}_{p}$, and let $\left\{\psi_{p}\left(\bar{x}_{p}, \bar{y}_{p}\right)\right\}_{p \in \omega}$ be a computable sequence of $\forall$-formulas, where the length of a tuple $\bar{y}_{p}$ is equal to the length of a tuple $\bar{c}_{p}$. We say that a system $\left\{\mathfrak{A}_{p}, \bar{c}_{p}, \psi_{p}\left(\bar{x}_{p}, \bar{y}_{p}\right)\right\}_{p \in \omega}$ is branching at level $p$ if, for any tuple $\bar{d}_{p}$ from $\mathfrak{A}$ with $\left(\mathfrak{A}, \bar{c}_{p}\right) \equiv_{1}\left(\mathfrak{A}, \bar{d}_{p}\right)$, the following two conditions hold:

(1) the set $\left\{\bar{b} \mid \mathfrak{A} \models \psi_{p}\left(\bar{b}, \bar{d}_{p}\right)\right\}$ is non-empty;

(2) if $\left\{\bar{b}_{i}\right\}_{i \in I}$ is some 1-1 enumeration of the set $\left\{\bar{b} \mid \mathfrak{A} \models \psi_{p}\left(\bar{b}, \bar{d}_{p}\right)\right\}$, where $I$ is an initial segment of $\omega$, and $\left\{\bar{a}_{i}\right\}_{i \in I}$ is a sequence of tuples from $\mathfrak{A}$ such that $\left(\mathfrak{A}, \bar{c}_{p}, \bar{a}_{0}, \ldots, \bar{a}_{i}\right) \equiv_{1}\left(\mathfrak{A}, \bar{d}_{p}, \bar{b}_{0}, \ldots, \bar{b}_{i}\right)$ for all $i \in I$, then there exists $n \in I$ with the following property:

$(*)$ there are infinitely many $t \geqslant p$ for which $\bar{a}_{0}, \ldots, \bar{a}_{n} \in \mathfrak{A}_{t}$, and there is an isomorphic embedding $\beta_{t}: \mathfrak{A}_{t} \rightarrow \mathfrak{A}_{t+1}$ such that $\mathfrak{A}_{t+1} \models \neg \psi_{p}\left(\beta_{t}\left(\bar{a}_{n}\right), \bar{c}_{p}\right)$, and $\beta_{t}$ is the identity on $\mathfrak{A}_{p}, \bar{a}_{0}, \ldots, \bar{a}_{n-1}$.

THEOREM 1 (on branching models [6]). If a system $\left\{\mathfrak{A}_{p}, \bar{c}_{p}, \psi_{p}\left(\bar{x}_{p}, \bar{y}_{p}\right)\right\}_{p \in \omega}$ is branching at any level $p \in \omega$, then the computable dimension of $\mathfrak{A}$ is effectively infinite.

Now we formulate the second version. Let $\mathfrak{A}$ be an infinite computable model for a language $L$, and let $\left\{\mathfrak{A}_{p}\right\}_{p \in \omega}$ be a computable sequence of finite models for $L$ such that $\mathfrak{A}_{p} \leq \mathfrak{A}_{p+1} \leq \mathfrak{A}$ for each $p$, and $\mathfrak{A}=\bigcup_{p} \mathfrak{A}_{p}$. Further, let $\left\{\psi_{p}^{n}\left(\bar{x}_{n}\right)\right\}_{p, n \in \omega}$ be a computable sequence of $\forall$-formulas, where $\bar{x}_{n}=\left\langle x^{0}, x^{1}, \ldots, x^{n}\right\rangle$. We say that a system $\left\{\mathfrak{A}_{p}, \psi_{p}^{n}\left(\bar{x}_{n}\right)\right\}_{p, n \in \omega}$ is branching at level $p$ if the following two conditions hold:

(1) the set $\left\{\bar{b} \mid \mathfrak{A} \models \psi_{p}^{n}(\bar{b}), n \in \omega, \bar{b}=\left\langle b^{0}, \ldots, b^{n}\right\rangle\right\}$ is non-empty;

(2) if $\left\{\bar{b}_{i}\right\}_{i \in I}$ is some 1-1 enumeration of the set $\left\{\bar{b} \mid \mathfrak{A} \models \psi_{p}^{n}(\bar{b}), n \in \omega, \bar{b}=\left\langle b^{0}, \ldots, b^{n}\right\rangle\right\}$, where $I$ is an initial segment of $\omega$, and $\left\{\bar{a}_{i}\right\}_{i \in I}$ is a sequence of tuples from $\mathfrak{A}$ such that $\left(\mathfrak{A}, \bar{a}_{0}, \ldots, \bar{a}_{i}\right) \equiv_{1}\left(\mathfrak{A}, \bar{b}_{0}, \ldots, \bar{b}_{i}\right)$ for all $i \in I$, then there exists $r \in I$ such that $\bar{a}_{r}=\left\langle a_{r}^{0}, a_{r}^{1}, \ldots, a_{r}^{n}\right\rangle$, and

$(*)$ there are infinitely many $t \geqslant p$ for which $\bar{a}_{0}, \ldots, \bar{a}_{r} \in \mathfrak{A}_{t}$, and there is an isomorphic embedding $\beta_{t}: \mathfrak{A}_{t} \rightarrow \mathfrak{A}_{t+1}$ such that $\mathfrak{A}_{t+1} \models \neg \psi_{p}^{n}\left(\beta_{t}\left(\bar{a}_{r}\right)\right)$, and $\beta_{t}$ is the identity on $\mathfrak{A}_{p}, \bar{a}_{0}, \ldots, \bar{a}_{r-1}$.

A proof of the previous theorem, offered in [6], implies that the present theorem admits the following modification.

THEOREM 2 (on branching models). If a system $\left\{\mathfrak{A}_{p}, \psi_{p}^{n}\left(\bar{x}_{n}\right)\right\}_{n, p \in \omega}$ is branching at any level $p \in \omega$, then the computable dimension of $\mathfrak{A}$ is effectively infinite.

\section{KRUSKAL'S THEOREM FOR $I$-TREES}

In what follows, we will need the ability to embed some finite $I$-trees in other ones. For this goal to be met, the well-known Kruskal theorem must be modified so as to yield a version tailored to the case of $I$-trees. Below is the exact formulation of Kruskal's theorem for finite trees with labelling function.

A quasiordering is a set $Q$ together with a reflexive transitive relation $\leqslant$. A well quasiordering (wqo) is a quasiordering $Q$ with the property that for any infinite sequence $\left\{q_{k} \mid k \in \omega\right\}$ of elements $q_{k} \in Q$, there exist indices $i$ and $j$ such that $i<j$ and $q_{i} \leqslant q_{j}$.

Let $\mathbb{T}$ be the set of all finite trees (up to isomorphism of trees). If $Q$ is an arbitrary quasiordering, we set

$$
\mathbb{T}(Q)=\{(T, l) \mid T \in \mathbb{T}, l: T \rightarrow Q\} .
$$

Thus an element of $\mathbb{T}(Q)$ is a finite tree with labels from $Q$. The function $l: T \rightarrow Q$ is called a labelling function. We write $\left(T_{1}, l_{1}\right) \leqslant\left(T_{2}, l_{2}\right)$ if there exists a one-to-one mapping $f: T_{1} \rightarrow T_{2}$ such that:

(1) $f(a \wedge b)=f(a) \wedge f(b)$ for all $a, b \in T_{1}$;

(2) $l_{1}(a) \leqslant l_{2}(f(a))$ for all $a \in T_{1}$. 
Obviously, $\mathbb{T}(Q)$ is quasiordered by this relation.

Kruskal's THEOREM. If $Q$ is an arbitrary wqo, then $\mathbb{T}(Q)$ is also a wqo.

Proof. See [7, 8]. From Kruskal's theorem we derive the following:

LEMMA 3. Let $\left\{\left(T_{i}, I_{i}\right) \mid i \in \omega\right\}$ be an infinite collection of finite $I$-trees, each with a labelling $l_{i}: T_{i} \rightarrow \omega$. Then there exist $i, j \in \omega, i<j$, and an embedding $f:\left(T_{i}, I_{i}\right) \rightarrow\left(T_{j}, I_{j}\right)$ such that for every $x \in T_{i}, l_{i}(x) \leqslant l_{j}(f(x))$.

Proof. We may assume that every $I_{i}$ is non-empty. (In other words, for every $i \in \omega$ the root of $T_{i}$ lies in $I_{i}$.) Otherwise, the subset $J=\left\{i \in \omega \mid I_{i}=\varnothing\right\}$ of indices is non-empty. If $J$ is finite, we consider the infinite collection $\left\{\left(T_{i}, I_{i}\right) \mid i \notin J\right\}$ instead of $\left\{\left(T_{i}, I_{i}\right) \mid i \in \omega\right\}$. If $J$ is infinite, we apply Kruskal's theorem immediately to the infinite collection $\left\{\left(T_{i}, I_{i}\right) \mid i \in J\right\}$.

Consider an infinite collection $\left\{I_{i} \mid i \in \omega\right\}$ of finite non-empty trees. For each $i \in \omega$, the labelling function $m_{i}: I_{i} \rightarrow \mathbb{T}(\omega) \times \omega$ on the tree $I_{i}$ is defined as follows: for any $x \in I_{i}$, we set $m_{i}(x)=\left(m_{i}^{1}(x), m_{i}^{2}(x)\right)$, where

(1) $m_{i}^{1}(x)=\left(S_{i}(x), l_{i} \uparrow S_{i}(x)\right) \in \mathbb{T}(\omega)$ with finite tree

$$
S_{i}(x)=\{x\} \cup\left\{y \in T_{i} \mid y \succ x \& \forall z \preccurlyeq y\left(x \prec z \rightarrow z \notin I_{i}\right)\right\}
$$

and a labelling function $l_{i} \uparrow S_{i}(x): S_{i}(x) \rightarrow \omega$. (Here, $l_{i}\left\lceil S_{i}(x)\right.$ denotes the restriction of $l_{i}$ to $S_{i}(x)$.)

(2) $m_{i}^{2}(x)=l_{i}(x)$.

It is clear that $\omega$ under the ordinary partial order is a wqo. By Kruskal's theorem, $\mathbb{T}(\omega)$ is also a wqo. It follows that the Cartesian product $\mathbb{T}(\omega) \times \omega$ together with the componentwise quasiorder is a wqo. Again, by Kruskal's theorem $\mathbb{T}(\mathbb{T}(\omega) \times \omega)$ is a wqo.

Thus, for the collection $\left\{\left(I_{i}, m_{i}\right) \mid i \in \omega\right\}$ of elements of $\mathbb{T}(\mathbb{T}(\omega) \times \omega)$, there exist $i$ and $j(i<j)$ with $\left(I_{i}, m_{i}\right) \leqslant\left(I_{j}, m_{j}\right)$, that is, there is an embedding $g: I_{i} \rightarrow I_{j}$ such that $m_{i}(x) \leqslant m_{j}(g(x))$ for every $x \in I_{i}$. The last inequality implies the following two conditions:

(1) there exists an embedding $h_{x}: S_{i}(x) \rightarrow S_{j}(g(x))$ such that $l_{i}(y) \leqslant l_{j}\left(h_{x}(y)\right)$ for every $y \in S_{i}(x)$ (since $\left.m_{i}^{1}(x) \leqslant m_{j}^{1}(g(x))\right)$;

(2) $l_{i}(x) \leqslant l_{j}(g(x))\left(\right.$ since $\left.m_{i}^{2}(x) \leqslant m_{j}^{2}(g(x))\right)$.

We define a mapping $f: T_{i} \rightarrow T_{j}$ as follows:

$$
f(y)= \begin{cases}g(y) & \text { if } y \in I_{i} \\ h_{x}(y) & \text { if } y \notin I_{i} \text { and } y \in S_{i}(x) \text { for some } x \in I_{i}\end{cases}
$$

Since $T_{i}=\bigcup_{x \in I_{i}} S_{i}(x)$, and $S_{i}\left(x_{1}\right) \cap S_{i}\left(x_{2}\right)=\varnothing$ for $x_{1} \neq x_{2}, f$ is well defined. It is easy to see that $f:\left(T_{i}, I_{i}\right) \rightarrow\left(T_{j}, I_{j}\right)$ is the desired embedding.

LEMMA 4. Let $\left\{\left(T_{i}, I_{i}\right) \mid i \in \omega\right\}$ be an infinite collection of finite $I$-trees. Then there exist $i, j \in \omega$, $i<j$, such that $\left(T_{i}, I_{i}\right)$ can be embedded in $\left(T_{j}, I_{j}\right)$.

The proof follows from Lemma 3 (we need only neglect the labelling functions).

LEMMA 5. Let $\left\{\left(T_{i}, I_{i}\right) \mid i \in \omega\right\}$ be an infinite collection of $I$-trees. (These trees need not to be finite, nor even finitely branching.) Then there exists an $i \in \omega$ such that for every finite subtree $T \subseteq T_{i}$, there is $j>i$ for which $\left(T, T \cap I_{i}\right)$ embeds in $\left(T_{j}, I_{j}\right)$.

Proof. Suppose that $\left\{\left(T_{i}, I_{i}\right) \mid i \in \omega\right\}$ is the collection of $I$-trees contradicting the statement of the lemma. Then, for each $i$, we would have some finite subtree $S_{i} \subseteq T_{i}$ such that $\left(S_{i}, S_{i} \cap I_{i}\right)$ did not embed 
in any $\left(T_{j}, I_{j}\right)$ with $j>i$. In particular, $\left(S_{i}, S_{i} \cap I_{i}\right)$ would not embed in $\left(S_{j}, S_{j} \cap I_{j}\right)$, for all $i, j(i<j)$. Thus the collection $\left\{\left(S_{i}, S_{i} \cap I_{i}\right) \mid i \in \omega\right\}$ contradicts Lemma 4.

LEMMA 6. Let $\left\{\left(T_{i}, I_{i}\right) \mid i \in \omega\right\}$ be as in Lemma 5. Then there is an $n \in \omega$ such that for every $i>n$ and every finite subtree $T \subseteq T_{i}$, there exists $j>i$ such that $\left(T, T \cap I_{i}\right)$ embeds in $\left(T_{j}, I_{j}\right)$.

Proof. If not, then we could find in $\omega$ an increasing sequence $i_{0}<i_{1}<i_{2}<\ldots$ such that $\left\{\left(T_{i_{k}}, I_{i_{k}}\right) \mid\right.$ $k \in \omega\}$ contradicted Lemma 5 .

LEMMA 7. Let $\left\{\left(T_{i}, I_{i}\right) \mid i \in \omega\right\}$ be as in Lemma 5. Then there is an $n \in \omega$ such that for every $i>n$ and every finite partial subordering $T \subseteq T_{i}$, there exists $j>i$ for which $\left(T, T \cap I_{i}\right)$ embeds in $\left(T_{j}, I_{j}\right)$.

Proof. Note that $T \subseteq T_{i}$ is a tree iff $T$ has a root. Thus, if $T$ has no root, we can consider a finite subtree $T^{\prime}=T \cup\left\{r_{i}\right\}$, where $r_{i}$ is a root of $T_{i}$. By Lemma 6 , there exists an embedding $h^{\prime}:\left(T^{\prime}, T^{\prime} \cap I_{i}\right) \rightarrow\left(T_{j}, I_{j}\right)$, for some $j>i$. Obviously, the restricted mapping $h=h^{\prime} \uparrow T$ is the desired embedding.

LEMMA 8. Let $\left\{\left(T_{i}, I_{i}\right) \mid i \in \omega\right\}$ be an infinite collection of finite $I$-trees. Then there is a number $m \in \omega$ such that for every index $i$ and every node $x \in T_{i}$ with level $_{T_{i}}(x)=m$, there exists an embedding $f:\left(T_{i}, I_{i}\right) \rightarrow\left(T_{j}, I_{j}\right), j>i$, for which

$$
\operatorname{level}_{T_{j}}(f(x))>\operatorname{level}_{T_{i}}(x) .
$$

Proof. Assume the contrary. Then, for every $m$, we would have an index $i_{m}$ and a node $x_{m} \in T_{i_{m}}$ with $\operatorname{level}_{T_{i_{m}}}\left(x_{m}\right)=m$ satisfying the following condition:

$(*)$ for each $j>i_{m}$ and for any embedding $f:\left(T_{i_{m}}, I_{i_{m}}\right) \rightarrow\left(T_{j}, I_{j}\right)$, we have $\operatorname{level}_{T_{j}}\left(f\left(x_{m}\right)\right)=$ level $_{T_{i_{m}}}\left(x_{m}\right)$.

Now the set $\left\{i_{0}, i_{1}, i_{2}, \ldots\right\}$ will be infinite, since each $T_{i}$ has finite height. Moreover, the index $i_{m}$ satisfies (*) not only for $x_{m}$ but also for all predecessors of $x_{m}$. Therefore we can choose $i_{m+1}>i_{m}$ for all $m$.

For each $m$, define the labelling function $l_{m}: T_{i_{m}} \rightarrow \omega$ on the $I$-tree $\left(T_{i_{m}}, I_{i_{m}}\right)$ by setting

$$
l_{m}(x)= \begin{cases}0 & \text { if } \text { level }_{T_{i_{m}}}(x)<m \\ 1 & \text { otherwise }\end{cases}
$$

Then $l_{m}\left(x_{m}\right)=1$ for all $m$.

However, for any $k, m(k>m)$ and for any embedding $f:\left(T_{i_{m}}, I_{i_{m}}\right) \rightarrow\left(T_{i_{k}}, I_{i_{k}}\right)$, we have

$$
\operatorname{level}_{T_{i_{k}}}\left(f\left(x_{m}\right)\right)=\operatorname{level}_{T_{i_{m}}}\left(x_{m}\right)=m<k .
$$

This forces $l_{k}\left(f\left(x_{m}\right)\right)=0$. Thus the sequence $\left\{\left(T_{i_{m}}, I_{i_{m}}\right) \mid m \in \omega\right\}$ contradicts Lemma 3 .

LEMMA 9. Let $\left\{\left(T_{i}, I_{i}\right) \mid i \in \omega\right\}$ be as in Lemma 8. Then there is a number $m \in \omega$ such that for every index $i$ and every node $y \in T_{i}$ with $\operatorname{level}_{T_{i}}(y) \geqslant m$, there exists an embedding $f:\left(T_{i}, I_{i}\right) \rightarrow\left(T_{j}, I_{j}\right)$, $j>i$, for which

$$
\operatorname{level}_{T_{j}}(f(y))>\operatorname{level}_{T_{i}}(y) .
$$

Proof. For every $y \in T_{i}$ with $\operatorname{level}_{T_{i}}(y) \geqslant m$, we find a node $x \preccurlyeq y$ in $T_{i}$ such that $\operatorname{level}_{T_{i}}(x)=m$, and then we apply Lemma 8 to that $x$.

LEMMA 10. Let $\left\{\left(T_{i}, I_{i}\right) \mid i \in \omega\right\}$ be any collection of $I$-trees. Then there exist an $n$ and an $m$ with the property that for all indices $i>n$, for every finite subtree $S \subseteq T_{i}$, and for any node $x \in S$ with $\operatorname{level}_{S}(x) \geqslant m$, there is an embedding $g:\left(S, S \cap I_{i}\right) \rightarrow\left(T_{j}, I_{j}\right), j>i$, such that

$$
\operatorname{level}_{T_{j}}(g(x))>\operatorname{level}_{S}(x) .
$$


Proof. Assume the contrary. The negation of the statement is as follows:

$$
\begin{gathered}
(\forall n)(\forall m)(\exists i>n)\left(\text { there exists a finite } S \subseteq T_{i}\right)(\exists x \in S)\left[\operatorname{level}_{S}(x) \geqslant m \&\right. \\
\left.\left.(\forall j>i) \text { (for every embedding } g:\left(S, S \cap I_{i}\right) \rightarrow\left(T_{j}, I_{j}\right)\right)\left(\operatorname{level}_{T_{j}}(g(x))=\operatorname{level}_{S}(x)\right)\right] .
\end{gathered}
$$

We apply this negation first with $n=0$ and $m=0$, yielding an index $i_{0}>0$ and a node $x_{0}$ at level $\geqslant 0$ in some finite subtree $S_{0}$ of $T_{i_{0}}$. Inductively, then, we apply the negation with $n=i_{k}$ and $m=k+1$ to obtain an index $i_{k+1}>i_{k}$ and a corresponding node $x_{k+1}$ at level $\geqslant k+1$ in some finite subtree $S_{k+1}$ of $T_{i_{k+1}}$. From the negation, we see that for any $j>i_{k}$, every embedding of $\left(S_{k}, S_{k} \cap I_{i_{k}}\right)$ into $\left(T_{j}, I_{j}\right)$ fixes the level of $x_{k}$. For any $j>k$, in particular, every embedding of $\left(S_{k}, S_{k} \cap I_{i_{k}}\right)$ into $\left(S_{j}, S_{j} \cap I_{i_{j}}\right)$ fixes the level of $x_{k}$. Thus the sequence $\left\{\left(S_{k}, S_{k} \cap I_{i_{k}}\right) \mid k \in \omega\right\}$ contradicts Lemma 9 .

LEMMA 11. Let $(T, I)$ be an $I$-tree such that $T_{\text {ext }}$ is non-empty and finite-branching. Then, for any infinite path $\gamma$ through $T$, all but finitely many nodes $x \in \gamma$ have the property that for every finite subtree $S \subseteq T[x], \gamma$ contains a $y \succ x$ such that $(S, S \cap I)$ embeds in $(T[y], T[y] \cap I)$.

Proof. Assume the contrary. Then there exists an infinite path $\gamma$ through $T$ such that the set $U$ of nodes for which the conclusion of the lemma fails is infinite. We represent all elements of $U$ as an ascending chain $u_{0} \prec u_{1} \prec u_{2} \prec \ldots$ Now, for each $i$, there exists a finite subtree $S_{i} \subseteq T\left[u_{i}\right]$ such that $\left(S_{i}, S_{i} \cap I\right)$ does not embed in $(T[y], T[y] \cap I)$ for any $y \succ x$ with $y \in \gamma$. In particular, $\left(S_{i}, S_{i} \cap I\right)$ does not embed in any $\left(T\left[u_{j}\right], T\left[u_{j}\right] \cap I\right)$ with $j>i$. Thus the sequence $\left\{\left(T\left[u_{i}\right], T\left[u_{i}\right] \cap I\right) \mid i \in \omega\right\}$ contradicts Lemma 5 .

\section{TREES WITH $\omega$-BRANCHING NODES}

In this section, we prove that $I$-trees from some significant subclass cannot be computably categorical. Let $(T, I)$ be a fixed computable $I$-tree with height $\omega$, which is $\omega$-branching at a node $x_{0}$, that is, $x_{0}$ has infinitely many immediate successors $x_{1}, x_{2}, \ldots$ We define the limit-supremum of a sequence $\left\{h t\left(T\left[x_{i}\right]\right) \mid\right.$ $i \in \omega\}$ to be

$$
\limsup _{i} \operatorname{ht}\left(T\left[x_{i}\right]\right)=\inf _{j} \sup _{i>j} \operatorname{ht}\left(T\left[x_{i}\right]\right) .
$$

Assume further that $\limsup _{i} \operatorname{su}\left(T\left[x_{i}\right]\right)=\omega$. Hence either infinitely many $T\left[x_{i}\right]$ have height $\omega$, or there exist trees $T\left[x_{i}\right]$ of arbitrarily large finite height.

Proposition 12. Let $(T, I)$ be a computable $I$-tree of height $\omega$ containing an $\omega$-branching node $x_{0}$ with immediate successors $x_{1}, x_{2}, \ldots$ such that

$$
\limsup _{i} h t\left(T\left[x_{i}\right]\right)=\omega .
$$

Then the computable dimension of $(T, I)$ is effectively infinite.

Proof. We may assume the universe of $T$ to be $\omega$. A successor tree of $x_{0}$ is a tree of the form $T\left[x_{i}\right]$ with $i \geqslant 1$. Lemma 10, applied to the collection $\left\{\left(T\left[x_{i}\right], T\left[x_{i}\right] \cap I\right) \mid i \geqslant 1\right\}$ of all successor trees, yields $m$ and $n$ in $\omega$ such that for every finite subtree $S \subseteq T\left[x_{i}\right]$, where $i>n$, and every node $x \in S$ with level $_{S}(x) \geqslant m$, there is an embedding of $(S, S \cap I)$ into some $\left(T\left[x_{j}\right], T\left[x_{j}\right] \cap I\right)$, where $j>i$, which maps $x$ to a node of greater level. We fix these values of $m$ and $n$ for the rest of the proof.

Let $\left\{T_{t} \mid t \in \omega\right\}$ be the preliminary representation for an $I$-tree $(T, I)$, where $T_{t}=\left\{r, x_{0}, x_{1}, \ldots, x_{n}\right\} \cup$ $\{0,1, \ldots, t\}$ is an $I$-tree under $\prec$ with distinguished initial subtree $T_{t} \cap I(r$ is the root of $T$ ). We define 
an increasing unbounded computable function $f(s)$ and a new representation $\left\{D_{s} \mid s \in \omega\right\}$ for $(T, I)$ in the following way.

At stage 0 , put $f(0)=0$ and $D_{0}=T_{0}$.

At stage $s+1$, we have $f(s)$ defined, and $D_{s}=T_{f(s)}$. We will say that a finite subtree $S \subseteq D_{s}$ is a successor tree at stage $s$ if $S$ is a tree of the form $D_{s}[y]$, where $y$ is an immediate successor of $x_{0}$ in $D_{s}$ (although not necessarily in $T$ ). Let $S_{1}, \ldots, S_{k}$ be the list of all successor trees at stage $s$ such that for each $l, 1 \leqslant l \leqslant k$, the tree $S_{l}$ differs from successor trees $D_{s}\left[x_{1}\right], \ldots, D_{s}\left[x_{n}\right]$, and $\operatorname{ht}\left(S_{l}\right) \geqslant m+1$.

We search for the least $t>f(s)$ such that for each $l, 1 \leqslant l \leqslant k$, and for any node $x \in S_{l}$ with level $_{S_{l}}(x) \geqslant m$, there exists a node $z \in T_{t}$ satisfying the following three conditions:

(1) $z$ is an immediate successor of $x_{0}$ in $T_{t}$;

(2) $T_{t}[z] \cap D_{s}=\varnothing$;

(3) there is an $I$-embedding $g: S_{l} \rightarrow T_{t}[z]$ with

$$
\operatorname{level}_{T_{t}}(g(x))>\operatorname{level}_{D_{s}}(x) .
$$

Then we put $f(s+1)=t$ and $D_{s+1}=T_{t}$.

We now prove that at each stage $s+1$, the desired $t$ exists. Consider an arbitrary $l$ such that $1 \leqslant l \leqslant k$, and any node $x \in S_{l}$ with $\operatorname{level}_{S_{l}}(x) \geqslant m$. Obviously, $S_{l} \subseteq T\left[x_{i}\right]$ for some unique $i>n$. Therefore there exists a sufficiently great $j>i$ such that $T\left[x_{j}\right] \cap D_{s}=\varnothing$, and there is an $I$-embedding $g_{l, x}: S_{l} \rightarrow T\left[x_{j}\right]$ with

$$
\operatorname{level}_{T\left[x_{j}\right]}\left(g_{l, x}(x)\right)>\operatorname{level}_{S_{l}}(x) .
$$

We denote this $j$ by $j(l, x)$.

Since $D_{s}$ is finite and $\mathrm{ht}(T)=\omega$, there exists a $t_{0}>f(s)$ such that

$$
\begin{gathered}
\left\{x_{j(l, x)} \mid 1 \leqslant l \leqslant k, x \in S_{l}, \operatorname{level}_{S_{l}}(x) \geqslant m\right\} \subseteq T_{t_{0}}, \\
\bigcup\left\{g_{l, x}\left(S_{l}\right) \mid 1 \leqslant l \leqslant k, x \in S_{l}, \operatorname{level}_{S_{l}}(x) \geqslant m\right\} \subseteq T_{t_{0}},
\end{gathered}
$$

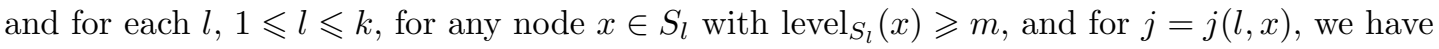

$$
\operatorname{level}_{T\left[x_{j}\right]}\left(g_{l, x}(x)\right)=\operatorname{level}_{T_{t_{0}}\left[x_{j}\right]}\left(g_{l, x}(x)\right) .
$$

Again consider an arbitrary $l$ such that $1 \leqslant l \leqslant k$, and any node $x \in S_{l}$ with $\operatorname{level}_{S_{l}}(x) \geqslant m$. By the choice of $j=j(l, x)$ and $t_{0}$, we conclude that $x_{j} \in T_{t_{0}}$ and $x_{j}$ is an immediate successor of $x_{0}$ in $T_{t_{0}}$. Therefore condition (1) is satisfied. Further, $T_{t_{0}}\left[x_{j}\right] \cap D_{s} \subseteq T\left[x_{j}\right] \cap D_{s}=\varnothing$, and so (2) is satisfied. Finally, there is an $I$-embedding

$$
g=g_{l, x}: S_{l} \rightarrow g_{l, x}\left(S_{l}\right) \subseteq T_{t_{0}}\left[x_{j}\right]
$$

which gives rise to the following chain of inequalities:

$$
\begin{gathered}
\operatorname{level}_{D_{s}}(x)=\operatorname{level}_{D_{s}}\left(x_{0}\right)+\operatorname{level}_{S_{l}}(x)+1<\operatorname{level}_{D_{s}}\left(x_{0}\right)+\operatorname{level}_{T\left[x_{j}\right]}(g(x))+1 \leqslant \\
\operatorname{level}_{T_{t_{0}}}\left(x_{0}\right)+\operatorname{level}_{T_{t_{0}}\left[x_{j}\right]}(g(x))+1=\operatorname{level}_{T_{t_{0}}}(g(x)) .
\end{gathered}
$$

Thus condition (3) is satisfied.

We apply Theorem 1 to the model $(T, I)$. For each $p \in \omega$, let $c_{1}, \ldots, c_{k_{p}}$ be the set of all immediate successors of $x_{0}$ in $D_{p}$. We put $\bar{c}_{p}=\left\langle c_{0}, c_{1}, \ldots, c_{k_{p}}\right\rangle$, where $c_{0}=x_{0}$. Also define the $\forall$-formula

$$
\psi_{p}\left(u^{0}, \ldots, u^{m+1}, w_{0}, \ldots, w_{k_{p}}\right)=\left(w_{0}=u^{0} \prec u^{1} \prec \ldots \prec u^{m+1}\right) \&
$$




$$
\forall y\left(u^{0} \preccurlyeq y \preccurlyeq u^{m+1} \rightarrow\left(y=u^{0} \vee \ldots \vee y=u^{m+1}\right)\right) \& \bigwedge_{i=1}^{k_{p}} \neg\left(u^{1} \preccurlyeq w_{i}\right) .
$$

Our goal is to prove that the system $\left\{\left(D_{p}, D_{p} \cap I\right), \bar{c}_{p}, \psi_{p}\left(\bar{u}, \bar{w}_{p}\right)\right\}_{p \in \omega}$ is branching at any level $p \in \omega$.

Let $p \in \omega$ and $\bar{d}_{p}=\left\langle d_{0}, d_{1}, \ldots, d_{k_{p}}\right\rangle$ be any tuple of elements of $T$ such that $\left(T, I, \bar{c}_{p}\right) \equiv_{1}\left(T, I, \bar{d}_{p}\right)$. There exists a tuple $\bar{a}=\left\langle a^{0}, \ldots, a^{m+1}\right\rangle$ for which $(T, I) \models \psi_{p}\left(\bar{a}, \bar{c}_{p}\right)$. To prove this, choose the least $q \geqslant n$ so that $D_{p}\left[x_{0}\right] \subseteq T\left[x_{1}\right] \cup \ldots \cup T\left[x_{q}\right]$. Since $D_{p}$ is finite, such $q$ must exist. Since $\operatorname{limsupht}_{i}\left(T\left[x_{i}\right]\right)=\omega$, there exists $i>q$ with $\operatorname{ht}\left(T\left[x_{i}\right]\right) \geqslant m+1$. Therefore there is a node $a^{m+1} \in T\left[x_{i}\right]$ with $\operatorname{level}_{T\left[x_{i}\right]}\left(a^{m+1}\right)=m$. Thus the chain $x_{0}=a^{0} \prec a^{1} \prec \ldots \prec a^{m+1}$ of all predecessors of $a^{m+1}$ in $T\left[x_{0}\right]$ has length $m+1$. By the choice of $i$, we conclude that $a^{1}=x_{i}$, and the successor tree $T\left[a^{1}\right]$ does not contain any element of $D_{p}$. Therefore $(T, I) \models \psi_{p}\left(a^{0}, \ldots, a^{m+1}, c_{0}, \ldots, c_{k_{p}}\right)$, and $\bar{a}=\left\langle a^{0}, \ldots, a^{m+1}\right\rangle$ is the desired tuple.

Now, let $z_{1}, \ldots, z_{\alpha}$ be all the elements of the finite set $\left\{z \in T \mid c_{0} \prec z \preccurlyeq c_{i}\right.$ for some $\left.i \leqslant k_{p}\right\}$. Obviously,

$$
\begin{gathered}
\left(T, I, \bar{c}_{p}\right) \models \exists z_{1} \ldots \exists z_{\alpha} \exists a^{0} \ldots \exists a^{m+1}\left(\left(c_{0}=a^{0} \prec a^{1} \prec \ldots \prec a^{m+1}\right) \&\right. \\
\left.\bigwedge_{i \neq j}\left(z_{i} \neq z_{j}\right) \& \bigwedge_{i=1}^{\alpha}\left(c_{0} \prec z_{i} \& \bigvee_{j=1}^{k_{p}}\left(z_{i} \preccurlyeq c_{j}\right)\right) \& \bigwedge_{i=1}^{\alpha} \neg\left(z_{i} \preccurlyeq a^{1}\right)\right) .
\end{gathered}
$$

Consequently, $\left(T, I, \bar{d}_{p}\right)$ insists on the similar property

$$
\begin{gathered}
\left(T, I, \bar{d}_{p}\right) \models \exists y_{1} \ldots \exists y_{\alpha} \exists v^{0} \ldots \exists v^{m+1}\left(\left(d_{0}=v^{0} \prec v^{1} \prec \ldots \prec v^{m+1}\right) \&\right. \\
\left.\bigwedge_{i \neq j}\left(y_{i} \neq y_{j}\right) \& \bigwedge_{i=1}^{\alpha}\left(d_{0} \prec y_{i} \& \bigvee_{j=1}^{k_{p}}\left(y_{i} \preccurlyeq d_{j}\right)\right) \& \bigwedge_{i=1}^{\alpha} \neg\left(y_{i} \preccurlyeq v^{1}\right)\right) .
\end{gathered}
$$

Let $d_{0}=b^{0} \prec b^{1} \prec \ldots \prec b^{m+1}$ be the chain of elements of $T$ such that $b^{m+1} \preccurlyeq v^{m+1}$ and $\operatorname{level}_{T\left[d_{0}\right]}\left(b^{m+1}\right)=m+1$. By the choice of elements $z_{1}, \ldots, z_{\alpha}$, and since $\left(T, I, \bar{c}_{p}\right) \equiv_{1}\left(T, I, \bar{d}_{p}\right)$, we see that there is no $d_{i}$ with $1 \leqslant i \leqslant k_{p}$ and $b^{1} \preccurlyeq d_{i}$. This immediately implies that $(T, I) \models \psi_{p}\left(b^{0}, \ldots, b^{m+1}\right.$, $\left.d_{0}, \ldots, d_{k_{p}}\right)$, and the set $\left\{\bar{b} \mid(T, I) \models \psi_{p}\left(\bar{b}, \bar{d}_{p}\right)\right\}$ is non-empty.

Now, let $\left\{\bar{b}_{j}\right\}_{j \in J}$ be some 1-1 enumeration of $\left\{\bar{b} \mid(T, I) \models \psi_{p}\left(\bar{b}, \bar{d}_{p}\right)\right\}$, where $J$ is an initial segment of $\omega$, and let $\left\{\bar{a}_{j}\right\}_{j \in J}$ be a sequence of tuples from $T$ such that $\left(T, I, \bar{c}_{p}, \bar{a}_{0}, \ldots, \bar{a}_{j}\right) \equiv_{1}\left(T, I, \bar{d}_{p}, \bar{b}_{0}, \ldots, \bar{b}_{j}\right)$, for each $j \in J$. Consider the first tuple $\bar{a}_{0}=\left\langle a_{0}^{0}, \ldots, a_{0}^{m+1}\right\rangle$. Since $(T, I) \models \psi_{p}\left(\bar{a}_{0}, \bar{c}_{p}\right)$, we see that $\operatorname{level}_{T\left[x_{0}\right]}\left(a_{0}^{m+1}\right)=m+1, a_{0}^{0}=x_{0}$, and $a_{0}^{1}=x_{i}$ for some $i>n$, and the successor tree $T\left[x_{i}\right]$ does not contain any element of $D_{p}$.

Choose a stage $s_{0}$ such that $D_{p} \cup\left\{\bar{a}_{0}\right\} \subseteq D_{s}$ and $\operatorname{level}_{D_{s}}\left(x_{0}\right)=\operatorname{level}_{T}\left(x_{0}\right)=k$, for all $s \geqslant s_{0}$. For any such $s$, we have level $D_{s}\left[x_{i}\right]\left(a_{0}^{m+1}\right)=m$. By construction, therefore, there exists $z \in D_{s+1}$ such that $z$ is an immediate successor of $x_{0}$ in $D_{s+1}, D_{s+1}[z] \cap D_{s}=\varnothing$, and there is an $I$-embedding $g: D_{s}\left[x_{i}\right] \rightarrow D_{s+1}[z]$ with the property

$$
\operatorname{level}_{D_{s+1}}\left(g\left(a_{0}^{m+1}\right)\right)>\operatorname{level}_{D_{s}}\left(a_{0}^{m+1}\right)=k+m+1 .
$$

For all such stages $s \geqslant s_{0}$ and for any $x \in D_{s}$, we define

$$
\beta_{s}(x)= \begin{cases}g(x) & \text { if } x \in D_{s}\left[x_{i}\right] \\ x & \text { if } x \in D_{s}-D_{s}\left[x_{i}\right]\end{cases}
$$


which is an $I$-embedding. In the successor tree $D_{s}\left[x_{i}\right]$ at stage $s$, there are no elements of $D_{p}$; so, we conclude that $\beta_{s}$ is identical on $D_{p}$. Finally, note that

$$
\operatorname{level}_{D_{s+1}}\left(\beta_{s}\left(a_{0}^{m+1}\right)\right)>k+m+1 .
$$

Therefore the chain $a_{0}^{0}=\beta_{s}\left(a_{0}^{0}\right) \prec \beta_{s}\left(a_{0}^{1}\right) \prec \ldots \prec \beta_{s}\left(a_{0}^{m+1}\right)$ does not contain all the nodes lying between $\beta_{s}\left(a_{0}^{0}\right)$ and $\beta_{s}\left(a_{0}^{m+1}\right)$ in $D_{s+1}$. Thus $\left(D_{s+1}, D_{s+1} \cap I\right) \models \neg \psi_{p}\left(\beta_{s}\left(\bar{a}_{0}\right), \bar{c}_{p}\right)$.

\section{TREES WITH INFINITE PATHS}

In this section, we assume that $T$ has an extendible node, that is, $T_{\text {ext }}$ is non-empty. We also think of $T_{\text {ext }}$ as being finite-branching, that is, any node $x \in T_{\text {ext }}$ has only finitely many extendible immediate successors in $T$.

The side tree above a node $x$ is denoted by $S[x]$, and is a subtree of $T[x]$ of the form

$$
S[x]=\left\{y \in T[x] \mid \forall z \in T\left(x \prec z \preccurlyeq y \rightarrow z \notin T_{\text {ext }}\right)\right\},
$$

where $x$ itself may or may not be extendible. Equivalently, we consider extendible immediate successors $x_{1}, x_{2}, \ldots$ of $x$. The side tree $S[x]$ is precisely $T[x]-\bigcup_{i} T\left[x_{i}\right]$. Thus $x$ is the only node of $S[x]$ which can be extendible in $T$, and $S[x]$ contains no infinite paths, although it can have height $\omega$ if it is infinite-branching.

Proposition 13. Let $(T, I)$ be a computable $I$-tree of height $\omega$ such that $T_{\text {ext }}$ is non-empty and finitebranching. If all side trees in $T$ have finite height, then the computable dimension of $(T, I)$ is effectively infinite.

Proof. We may assume that $T=\omega$. Fix some infinite path $\gamma$ lying in $T$. By Lemma 11, the set $U$ of all nodes in $\gamma$ for which the statement of the lemma fails is finite. Let $m=\max \left\{\operatorname{level}_{T}(x) \mid x \in U\right\}$. Then there exists $y \succ x$ with $y \in \gamma$ such that $(S, S \cap I)$ embeds in $(T[y], T[y] \cap I)$ for every node $x \in \gamma$ with $\operatorname{level}_{T}(x)>m$ and for each finite subtree $S \subseteq T[x]$.

Let $x_{m}$ be a node in $\gamma$ such that $\operatorname{level}_{T}(x)=m$, and let $r=x_{0} \prec x_{1} \prec \ldots \prec x_{m}$ be all the predecessors of $x_{m}$ in $T$. By $T_{s}$ we denote the subtree of $T$ with nodes $\left\{x_{0}, x_{1}, \ldots, x_{m}\right\} \cup\{0,1, \ldots, s\}$ under $\prec$ with distinguished initial subtree $T_{s} \cap I$. We define an increasing computable function $f(s)$ and a computable sequence $\left\{D_{s} \mid s \in \omega\right\}$ of finite subtrees of $T$ in the following way.

At stage 0 , put $f(0)=0$ and $D_{0}=T_{0}$.

At stage $s+1$, we have $f(s)$ defined, and $D_{s}=T_{f(s)}$. Put $l_{s}=\operatorname{ht}\left(D_{s}\right)>m$. For each $l, m<l<l_{s}$, let $\left\{v_{l, s}^{0}, v_{l, s}^{1}, \ldots, v_{l, s}^{n_{l, s}}\right\}$ be all the nodes in $D_{s}$ lying at level $l$ in $D_{s}$. We search for the least $t>f(s)$ so that for each $l, m<l<l_{s}$, one of the following two conditions holds:

(a) there exist $k \leqslant n_{l, s}$ and an $I$-embedding $g: D_{s}\left[v_{l, s}^{k}\right] \rightarrow T_{t}\left[v_{l, s}^{k}\right]$ such that

$$
\operatorname{level}_{T_{t}}\left(g\left(v_{l, s}^{k}\right)\right) \geqslant \operatorname{level}_{D_{s}}\left(v_{l, s}^{k}\right)+s
$$

(b) there exists $x \in T_{t}$ such that level $T_{t}(x)=l$, ht $\left(T_{t}[x]\right) \geqslant s$, and either $x \notin D_{s}$ or $\operatorname{level}_{D_{s}}(x)<l$.

We put $f(s+1)=t$ and $D_{s+1}=T_{t}$.

We now prove that at each stage $s+1$, either condition (a) or condition (b) must hold for some $t$. Consider an arbitrary $l$ with $m<l<l_{s}$. Suppose that there exists an extendible node $x \in\left\{v_{l, s}^{0}, \ldots, v_{l, s}^{n_{l, s}}\right\}$. By the choice of $m$, the finite subtree $D_{s}[x]$ can be $I$-embedded in some $T[y]$ for $y \in \gamma, y \succ x$. By induction, $D_{s}[x]$ can be $I$-embedded in $T[x]$ with the root mapping to a node at an arbitrarily high level of $T$. Therefore 
there exist $t_{l}>f(s)$ and an $I$-embedding $g: D_{s}[x] \rightarrow T_{t_{l}}[x]$ such that $\operatorname{level}_{T_{t_{l}}}(g(x)) \geqslant \operatorname{level}_{D_{s}}(x)+s$, that is, condition (a) will hold for $x$.

Otherwise, none of $v_{l, s}^{0}, \ldots, v_{l, s}^{n_{l, s}}$ is extendible. Nevertheless some node $x$ at level $l$ in $T$ must be extendible. Therefore either $x \in D_{s}$ and $\operatorname{level}_{D_{s}}(x)<l$, or $x \notin D_{s}$. Then there exists $t_{l}>f(s)$ such that condition (b) will hold for $x$. Now we define the desired $t$ to be equal to $\max \left\{t_{l} \mid m<l<l_{s}\right\}$.

We apply Theorem 2 to the model $(T, I)$. For all $p, n \in \omega$, define the $\forall$-formula

$$
\begin{gathered}
\psi_{p}^{n}\left(x^{0}, x^{1}, \ldots, x^{n}\right)=\left(x^{0} \prec x^{1} \prec \ldots \prec x^{n}\right) \& \forall y\left(x^{0} \preccurlyeq y\right) \& \\
\forall y\left(x^{0} \preccurlyeq y \preccurlyeq x^{n} \rightarrow\left(y=x^{0} \vee \ldots \vee y=x^{n}\right)\right) .
\end{gathered}
$$

(The definition of $\psi_{p}^{n}\left(\bar{x}_{n}\right)$ does not depend on $p$.)

We prove that the system $\left\{\left(D_{p}, D_{p} \cap I\right), \psi_{p}^{n}\left(\bar{x}_{n}\right)\right\}_{p, n \in \omega}$ is branching at any level $p \in \omega$. Since $D_{p}$ is finite, we can define

$$
l=\max \left\{\operatorname{level}_{T}(x) \mid x \in D_{p}\right\}+1 .
$$

Note that $l>m$. Since all side trees in $T$ have finite height and $T_{\text {ext }}$ is finite-branching, we have

$$
l_{1}=\max \left\{\operatorname{ht}(S[x]) \mid x \in T_{\text {ext }}, \operatorname{level}_{T}(x) \leqslant l\right\} .
$$

Take a stage $s_{0}$ so that $s_{0} \geqslant \max \left\{p, l_{1}\right\}$ and $\left\{x \in T_{\text {ext }} \mid \operatorname{level}_{T}(x) \leqslant l\right\} \subseteq D_{s_{0}}$. Then, for any stage $s \geqslant s_{0}$, condition (a) will never again hold for any $k \leqslant n_{l, s}$ with $v_{l, s}^{k}$ non-extendible, and condition (b) will not hold for any non-extendible node $x$. Thus only finitely many extendible nodes $v_{l, s}^{k}$ satisfy either (a) or (b) at each stage $s \geqslant s_{0}$. But every extendible node $x$ at level $l$ in $T$ already satisfies level $D_{D_{s}}(x)=l$ at stage $s \geqslant s_{0}$. Therefore condition (b) will never hold again at stages $s \geqslant s_{0}$. Thus there must exist an extendible node $x$ at level $l$ in $T$ which satisfies condition (a) at infinitely many stages $s \geqslant s_{0}$.

By $y_{1}, \ldots, y_{\alpha}$ we denote all extendible nodes at level $l$ in $T$. By the above argument, we may assume that $y_{1}$ satisfies condition (a) at infinitely many stages $s \geqslant s_{0}$.

Let now $\left\{\bar{b}_{j}\right\}_{j \in J}$ be some 1-1 enumeration of a non-empty set $\left\{\bar{b} \mid(T, I) \models \psi_{p}^{n}(\bar{b}), \bar{b}=\left\langle b^{0}, \ldots, b^{n}\right\rangle\right\}$, where $J$ is an initial segment of $\omega$, and let $\left\{\bar{a}_{j}\right\}_{j \in J}$ be a sequence of tuples from $T$ such that $\left(T, I, \bar{a}_{0}, \ldots, \bar{a}_{j}\right) \equiv_{1}\left(T, I, \bar{b}_{0}, \ldots, \bar{b}_{j}\right)$ for each $j \in J$. It is clear that for any node $y_{i}$ with $1 \leqslant i \leqslant \alpha$, there is a tuple $\bar{b}=\left\langle b^{0}, \ldots, b^{n}\right\rangle$ such that $(T, I) \models \psi_{p}^{n}(\bar{b})$, and $y_{i}$ is an element of $\bar{b}$. Therefore there exists $q \in J$ such that all nodes $y_{1}, \ldots, y_{\alpha}$ have already appeared in tuples $\bar{b}_{0}, \ldots, \bar{b}_{q}$. By the choice of our elements $y_{1}, \ldots, y_{\alpha}$, and since $\left(T, I, \bar{a}_{0}, \ldots, \bar{a}_{q}\right) \equiv_{1}\left(T, I, \bar{b}_{0}, \ldots, \bar{b}_{q}\right)$, we conclude that all nodes $y_{1}, \ldots, y_{\alpha}$ must appear in tuples $\bar{a}_{0}, \ldots, \bar{a}_{q}$. In particular, the node $y_{1}$ appears in one of the tuples $\bar{a}_{0}, \ldots, \bar{a}_{q}$.

Take the least $r \in J$ such that $y_{1}$ has appeared in a tuple $\bar{a}_{r}=\left\langle a_{r}^{0}, a_{r}^{1}, \ldots, a_{r}^{n}\right\rangle$, that is, $n \geqslant l$ and $a_{r}^{l}=y_{1}$. Choose a stage $s_{1} \geqslant s_{0}$ so that $\bar{a}_{0}, \ldots, \bar{a}_{r} \in D_{s_{1}}$. By our choice of $y_{1}$, for infinitely many stages $s \geqslant s_{1}$, there is an $I$-embedding $g: D_{s}\left[y_{1}\right] \rightarrow D_{s+1}\left[y_{1}\right]$ with the property

$$
\operatorname{level}_{D_{s+1}}\left(g\left(y_{1}\right)\right) \geqslant \operatorname{level}_{D_{s}}\left(y_{1}\right)+s .
$$

For all such stages $s \geqslant s_{1}$, we define

$$
\beta_{s}(x)= \begin{cases}g(x) & \text { if } x \in D_{s}\left[y_{1}\right] \\ x & \text { if } x \in D_{s}-D_{s}\left[y_{1}\right]\end{cases}
$$

Then $\beta_{s}: D_{s} \rightarrow D_{s+1}$ is an $I$-embedding. Since level ${ }_{T}\left(y_{1}\right)=l$ and all nodes from $D_{p}$ lie below the level $l$ in the tree $D_{s}, \beta_{s}$ is identical on $D_{p}$. By the choice of $r$, the node $y_{1}$ is not an element of any tuple 
$\bar{a}_{0}, \ldots, \bar{a}_{r-1}$. Therefore $\beta_{s}$ is the identity on $\bar{a}_{0}, \ldots, \bar{a}_{r-1}$. Finally, we observe that

$$
\begin{gathered}
\operatorname{level}_{D_{s+1}}\left(\beta_{s}\left(a_{r}^{l}\right)\right) \geqslant \operatorname{level}_{D_{s}}\left(a_{r}^{l}\right)+s>\operatorname{level}_{D_{s}}\left(a_{r}^{l}\right)=l, \\
\operatorname{level}_{D_{s+1}}\left(\beta_{s}\left(a_{r}^{l-1}\right)\right)=\operatorname{level}_{D_{s+1}}\left(a_{r}^{l-1}\right)=l-1 .
\end{gathered}
$$

Thus $\left(D_{s+1}, D_{s+1} \cap I\right) \models \neg \psi_{p}^{n}\left(\beta_{s}\left(\bar{a}_{r}\right)\right)$.

\section{TREES OF HEIGHT EXCEEDING $\omega$}

We now prove that no $I$-tree of height exceeding $\omega$ is computably categorical. In such trees, there exists a node $x_{\omega}$ at level $\omega$. The predecessors of $x_{\omega}$ form a computable infinite chain in $T$. The chain is not a path, but it is still perfectly useful for our purposes. We will appeal to Kruskal's theorem again to guarantee the existence of the necessary embeddings upwards along this chain.

Proposition 14. Let $(T, I)$ be a computable $I$-tree with $h t(T)>\omega$. Then the computable dimension of $(T, I)$ is effectively infinite.

Proof. Since $\operatorname{ht}(T)>\omega, T$ contains a node $x_{\omega}$ at level $\omega$. Let $r=x_{0} \prec x_{1} \prec x_{2} \prec \ldots$ be all the predecessors of $x_{\omega}$ in $T$. For each $i \in \omega$, we set $S_{i}=T\left[x_{i}\right]-T\left[x_{i+1}\right]$, and for a limit index, define $S_{\omega}=T-\bigcup_{i \in \omega} S_{i}$. Note that $S_{i} \cap S_{j}=\varnothing$ for any $i, j \in \omega \cup\{\omega\}$ with $i \neq j$, and if $x \in S_{\omega}$, then $x_{i} \prec x$ and $\operatorname{level}_{T}(x) \geqslant \omega$, for any $i \in \omega$. In particular, $x_{\omega} \in S_{\omega}$.

We apply Lemma 7 to the collection of $I$-trees $\left(S_{i}, S_{i} \cap I\right), i \in \omega$, yielding an $n$ such that for every $i \geqslant n$ and every finite partial subordering $S \subseteq S_{i}$, there is some $j>i$ for which $(S, S \cap I)$ embeds in $\left(S_{j}, S_{j} \cap I\right)$. By induction, then, every finite subordering of each such $S_{i} I$-embeds in infinitely many $S_{j}$, where $j>i$.

Let $\left\{T_{s} \mid s \in \omega\right\}$ be the preliminary representation for an $I$-tree $(T, I)$, where $T_{s}=\left\{x_{0}, x_{1}, \ldots, x_{n}\right\} \cup$ $\{0,1, \ldots, s\} \cup\left\{x_{\omega}\right\}$ is an $I$-tree under $\prec$ with distinguished initial subtree $T_{s} \cap I$. For each $s \in \omega$, let

$$
\left\{x_{n}=x_{n, s} \prec x_{n+1, s} \prec \ldots \prec x_{l_{s}, s}\right\}
$$

be the chain of all the predecessors of $x_{\omega}$ in $T_{s}\left[x_{n}\right]$. Clearly, $\lim _{s} x_{i, s}=x_{i}$ for all $i$. We define an increasing unbounded computable function $f(s)$ and a new representation $\left\{D_{s} \mid s \in \omega\right\}$ for $(T, I)$ in the following way.

At stage 0 , put $f(0)=0$ and $D_{0}=T_{0}$.

At stage $s+1$, given $f(s)$ and $D_{s}=T_{f(s)}$ defined, we search for the least $t>f(s)$ satisfying the following condition:

$(*)$ for each $i$ with $n \leqslant i \leqslant l_{t}$, there exists an $I$-embedding $g_{i}: D_{s}\left[x_{i, t}\right] \rightarrow T_{t}\left[x_{i, t}\right]$ with the property

$$
\forall x \in D_{s}\left[x_{i, t}\right]\left(x_{l_{t}, t} \nprec x \rightarrow \operatorname{level}_{D_{s}}(x)<\operatorname{level}_{T_{t}}\left(g_{i}(x)\right)\right) \& \forall x \in D_{s}\left[x_{i, t}\right]\left(x_{l_{t}, t} \prec x \rightarrow g_{i}(x)=x\right) .
$$

Then we put $f(s+1)=t, D_{s+1}=T_{f(s)}$.

We observe that above we defined $D_{s}\left[x_{i, t}\right]$ to be $\left\{y \in D_{s} \mid x_{i, t} \preccurlyeq y\right\}$, and considered an $I$-embedding $g_{i}: D_{s}\left[x_{i, t}\right] \rightarrow T_{t}\left[x_{i, t}\right]$ as the embedding of the partial ordering $D_{s}\left[x_{i, t}\right]$ into $T_{t}\left[x_{i, t}\right]$ which preserves $I$.

We now prove that at each stage $s+1$, the desired $t$ exists. Since $D_{s}\left[x_{n}\right]$ is finite, there exists $m \geqslant n$ such that $S_{i} \cap D_{s}\left[x_{n}\right]=\varnothing$, for every $i>m, i \in \omega$. Therefore $D_{s}\left[x_{i}\right] \subseteq S_{\omega}$, for every $i>m$. Since each sequence $\left\{x_{i, t}\right\}_{t \in \omega}$ converges to $x_{i}$, we can find a stage $t_{0}>f(s)$ so that for all $t \geqslant t_{0}$,

$$
x_{n, t}=x_{n}, \ldots, x_{m, t}=x_{m}, \quad x_{m+1, t}=x_{m+1} .
$$

This implies that $D_{s}\left[x_{i, t}\right] \subseteq D_{s}\left[x_{i}\right] \subseteq S_{\omega}$ for all $t \geqslant t_{0}$ and for every $i>m$. 
Consider an arbitrary $i$ such that $n \leqslant i \leqslant m$. Obviously, the identity embedding $\iota: D_{s}\left[x_{i}\right] \rightarrow T_{t_{0}}\left[x_{i}\right]$ satisfies the following condition:

$$
\forall x \in D_{s}\left[x_{i}\right]\left(x_{l_{t_{0}}, t_{0}} \nprec x \rightarrow \operatorname{level}_{D_{s}}(x) \leqslant \operatorname{level}_{T_{t_{0}}}(\iota(x))\right) \& \forall x \in D_{s}\left[x_{i}\right]\left(x_{l_{t_{0}}, t_{0}} \prec x \rightarrow \iota(x)=x\right) .
$$

Since $T_{t_{0}}\left[x_{i}\right]$ is finite, there are only finitely many nodes $x_{i}=x_{i_{0}} \prec x_{i_{1}} \prec \ldots \prec x_{i_{q}}$ for which

$$
T_{t_{0}}\left[x_{i}\right] \cap S_{i_{0}} \neq \varnothing, \ldots, T_{t_{0}}\left[x_{i}\right] \cap S_{i_{q}} \neq \varnothing, \quad T_{t_{0}}\left[x_{i}\right]-\bigcup_{p \leqslant q} S_{i_{p}} \subseteq S_{\omega} .
$$

By the choice of $n$, we can find an $I$-embedding

$$
h_{0}: T_{t_{0}}\left[x_{i}\right] \cap S_{i_{0}} \rightarrow S_{j_{0}}
$$

where $j_{0}>i_{0}$, such that $x_{i_{0}} \in T_{t_{0}}\left[x_{i}\right] \cap S_{i_{0}}$ iff $x_{j_{0}} \in h_{0}\left(T_{t_{0}}\left[x_{i}\right] \cap S_{i_{0}}\right)$, and $h_{0}\left(x_{i_{0}}\right)=x_{j_{0}}$, if either is true.

Then we can find an $I$-embedding

$$
h_{1}: T_{t_{0}}\left[x_{i}\right] \cap S_{i_{1}} \rightarrow S_{j_{1}}
$$

where $j_{1}>j_{0}$, such that $x_{i_{1}} \in T_{t_{0}}\left[x_{i}\right] \cap S_{i_{1}}$ iff $x_{j_{1}} \in h_{1}\left(T_{t_{0}}\left[x_{i}\right] \cap S_{i_{1}}\right)$, and $h_{1}\left(x_{i_{1}}\right)=x_{j_{1}}$, if either is true, and so on.

Finally, we define the identity map

$$
h_{\omega}: T_{t_{0}}\left[x_{i}\right]-\bigcup_{p \leqslant q} S_{i_{p}} \rightarrow T_{t_{0}}\left[x_{i}\right]-\bigcup_{p \leqslant q} S_{i_{p}} .
$$

The union $f_{i}=h_{0} \cup \ldots \cup h_{q} \cup h_{\omega}$ of these $I$-embeddings is the $I$-embedding of $T_{t_{0}}\left[x_{i}\right]$ into $T\left[x_{i+1}\right]$.

Further, we can find a stage $t_{1}>t_{0}$ so that $\bigcup_{n \leqslant i \leqslant m} f_{i}\left(T_{t_{0}}\left[x_{i}\right]\right) \subseteq T_{t_{1}}$. Then our $I$-embedding $f_{i}$ is of the form $f_{i}: T_{t_{0}}\left[x_{i}\right] \rightarrow T_{t_{1}}\left[x_{i+1}\right]$, for every $i$. Now fix an arbitrary $i$ such that $n \leqslant i \leqslant l_{t_{1}}$. There are two cases to consider.

Suppose $n \leqslant i \leqslant m$. Take the following composition of $I$-embeddings:

$$
g_{i}=f_{i} \circ \iota: D_{s}\left[x_{i}\right] \rightarrow T_{t_{1}}\left[x_{i+1}\right] \subseteq T_{t_{1}}\left[x_{i}\right] .
$$

If $x \in D_{s}\left[x_{i}\right]$ and $x_{l_{t_{1}}, t_{1}} \prec x$, then $x \in S_{\omega}$. Therefore $g_{i}(x)=h_{\omega}(x)=x$. If $x \in D_{s}\left[x_{i}\right]$ and $x_{l_{t_{1}}, t_{1}} \nprec x$, then $x \in T_{t_{0}}\left[x_{i}\right] \cap S_{i_{p}}$ for some $p \leqslant q$. Therefore we obtain the following chain of inequalities:

$$
\begin{gathered}
\operatorname{level}_{D_{s}}(x) \leqslant \operatorname{level}_{T_{t_{0}}}(x)=\operatorname{level}_{T_{t_{0}}}\left(x_{i}\right)+\operatorname{level}_{T_{t_{0}}\left[x_{i}\right]}(x)< \\
\operatorname{level}_{T_{t_{1}}}\left(x_{i+1}\right)+\operatorname{level}_{T_{t_{1}}\left[x_{i+1}\right]}\left(f_{i}(x)\right)=\operatorname{level}_{T_{t_{1}}}\left(f_{i}(x)\right)=\operatorname{level}_{T_{t_{1}}}\left(g_{i}(x)\right) .
\end{gathered}
$$

Suppose $m<i \leqslant l_{t_{1}}$. Then $D_{s}\left[x_{i, t_{1}}\right] \subseteq D_{s}\left[x_{i}\right] \subseteq S_{\omega}$. Consequently, we have $x_{l_{t_{1}}, t_{1}} \prec x$ for any $x \in D_{s}\left[x_{i, t_{1}}\right]$. It is sufficient to take the identity map

$$
g_{i}=\mathrm{id}: D_{s}\left[x_{i, t_{1}}\right] \rightarrow T_{t_{1}}\left[x_{i, t_{1}}\right]
$$

to satisfy the desired conditions. Thus there exists a $t=t_{1}$ for which condition $(*)$ holds.

Again we apply Theorem 1 to the model $(T, I)$. Define the $\forall$-formula

$$
\psi\left(u^{0}, u^{1}, v\right)=\left(u^{0} \prec u^{1} \prec v\right) \& \forall y\left(u^{0} \preccurlyeq y \preccurlyeq u^{1} \rightarrow\left(y=u^{0} \vee y=u^{1}\right)\right) .
$$


Therefore $(T, I) \models \psi\left(a^{0}, a^{1}, x_{\omega}\right)$ iff $a^{0}$ and $a^{1}$ lie on our computable infinite chain under $x_{\omega}$, and $a^{0}$ is an immediate predecessor of $a^{1}$ in $T$. We will prove that the system $\left\{\left(D_{p}, D_{p} \cap I\right), x_{\omega}, \psi\left(u^{0}, u^{1}, v\right)\right\}_{p \in \omega}$ is branching at any level $p \in \omega$. (Formula $\psi$ and parameter $x_{\omega}$ do not depend on $p$, and are the same for all $p$.)

Let $p \in \omega$ and $y_{\omega}$ be any element of $T$ such that $\left(T, I, x_{\omega}\right) \equiv_{1}\left(T, I, y_{\omega}\right)$. Since $x_{\omega}$ has infinite level and $\left(T, I, x_{\omega}\right) \equiv_{1}\left(T, I, y_{\omega}\right), y_{\omega}$ also lies on the infinite level, that is, level $_{T}\left(y_{\omega}\right) \geqslant \omega$, and there exists a countable chain

$$
r=y_{0} \prec y_{1} \prec y_{2} \prec \ldots
$$

of all the predecessors of $y_{\omega}$ sitting at finite levels in $T$. Therefore, for every $i \in \omega,\left\langle y_{i}, y_{i+1}\right\rangle \in\{\bar{b} \mid(T, I) \models$ $\left.\psi\left(\bar{b}, y_{\omega}\right)\right\}$. Thus the set $\left\{\bar{b} \mid(T, I) \models \psi\left(\bar{b}, y_{\omega}\right)\right\}$ is not empty.

Let now $\left\{\bar{b}_{j}\right\}_{j \in J}$ be some 1-1 enumeration for the set $\left\{\bar{b} \mid(T, I) \models \psi\left(\bar{b}, y_{\omega}\right)\right\}$, where $J$ is an initial segment of $\omega$, and let $\left\{\bar{a}_{j}\right\}_{j \in J}$ be a sequence of pairs from $T$ such that $\left(T, I, x_{\omega}, \bar{a}_{0}, \ldots, \bar{a}_{j}\right) \equiv_{1}\left(T, I, y_{\omega}, \bar{b}_{0}, \ldots, \bar{b}_{j}\right)$, for all $j \in J$. Since $D_{p}$ is finite, there exists the natural

$$
m=\max \left\{k \in \omega \mid k \geqslant n \& \exists y \in D_{p}\left[x_{n}\right]\left(y \notin S_{\omega} \& x_{k} \preccurlyeq y\right)\right\} .
$$

Thus, for every $i \geqslant m+1$, the tree $T\left[x_{i}\right]$ contains no element of $D_{p}\left[x_{n}\right]-S_{\omega}$. As noted above, $\left\langle y_{m}, y_{m+1}\right\rangle \in$ $\left\{\bar{b} \mid(T, I) \models \psi\left(\bar{b}, y_{\omega}\right)\right\}$. Then $\left\langle y_{m}, y_{m+1}\right\rangle=\bar{b}_{j}=\left\langle b_{j}^{0}, b_{j}^{1}\right\rangle$ for some $j \in J$, and we conclude that $T \models$ $\exists z_{0} \ldots \exists z_{m}\left(z_{0} \prec \ldots \prec z_{m}=b_{j}^{0}\right)$. Therefore we must have $T \models \exists z_{0} \ldots \exists z_{m}\left(z_{0} \prec \ldots \prec z_{m}=a_{j}^{0}\right)$. Hence there exists $j \in J$ with $\operatorname{level}_{T}\left(a_{j}^{0}\right) \geqslant m$.

Consider the least $j \in J$ such that $\operatorname{level}_{T}\left(a_{j}^{0}\right) \geqslant m$ for the pair $\bar{a}_{j}=\left\langle a_{j}^{0}, a_{j}^{1}\right\rangle$. It follows that $a_{j}^{0}=x_{i}$ and $a_{j}^{1}=x_{i+1}$, where $i=\operatorname{level}_{T}\left(a_{j}^{0}\right) \geqslant m \geqslant n$. In particular, the tree $T\left[x_{i+1}\right]$ contains no elements of $D_{p}\left[x_{n}\right]-S_{\omega}$. Choose a stage $s_{0}$ so that $D_{p} \cup\left\{\bar{a}_{0}, \ldots, \bar{a}_{j}\right\} \subseteq D_{s}$, and $\operatorname{level}_{D_{s}}\left(a_{j}^{0}\right)=\operatorname{level}_{T}\left(a_{j}^{0}\right)$ for all $s \geqslant s_{0}$. For any such $s$, we have $a_{j}^{1}=x_{i+1}=x_{i+1, f(s)}$. By construction, therefore, there exists an $I$-embedding $g: D_{s}\left[x_{i+1}\right] \rightarrow D_{s+1}\left[x_{i+1}\right]$ with the property

$$
\begin{gathered}
\operatorname{level}_{D_{s}}\left(x_{i+1}\right)<\operatorname{level}_{D_{s+1}}\left(g\left(x_{i+1}\right)\right), \\
\forall x \in D_{s}\left[x_{i+1}\right]\left(x_{l_{f(s+1)}, f(s+1)} \prec x \rightarrow g(x)=x\right) .
\end{gathered}
$$

For all such stages $s \geqslant s_{0}$, define

$$
\beta_{s}(x)= \begin{cases}g(x) & \text { if } x \in D_{s}\left[x_{i+1}\right] \\ x & \text { if } x \in D_{s}-D_{s}\left[x_{i+1}\right],\end{cases}
$$

which is an $I$-embedding. Since $T\left[x_{i+1}\right]$ contains no elements of $D_{p}\left[x_{n}\right]-S_{\omega}$, we have $D_{p}-S_{\omega} \subseteq D_{s}-$ $D_{s}\left[x_{i+1}\right]$, and so $\beta_{s}$ is identical on $D_{p}-S_{\omega}$. Besides, by the choice of $g, \beta_{s}$ is identical on $D_{p} \cap S_{\omega}$. Also, by the choice of $j \in J$, all the previous tuples $\bar{a}_{0}, \ldots, \bar{a}_{j-1}$ do not lie in $D_{s}\left[x_{i+1}\right]$. This implies that $\beta_{s}$ is identical on elements of the tuples $\bar{a}_{0}, \ldots, \bar{a}_{j-1}$. Finally, we observe that

$$
\operatorname{level}_{D_{s+1}}\left(\beta_{s}\left(a_{j}^{1}\right)\right)>\operatorname{level}_{D_{s}}\left(a_{j}^{1}\right)=\operatorname{level}_{D_{s}}\left(a_{j}^{0}\right)+1=\operatorname{level}_{D_{s+1}}\left(\beta_{s}\left(a_{j}^{0}\right)\right)+1 \text {. }
$$

Therefore there exists $y \in D_{s+1}$ for which $\beta_{s}\left(a_{j}^{0}\right) \prec y \prec \beta_{s}\left(a_{j}^{1}\right)$. Thus $\left(D_{s+1}, D_{s+1} \cap I\right) \models \neg \psi\left(\beta_{s}\left(a_{j}^{0}\right)\right.$, $\left.\beta_{s}\left(a_{j}^{1}\right), x_{\omega}\right)$. 


\section{TREES OF INFINITE HEIGHT}

We now prove the basic theorem for $I$-trees of infinite height.

THEOREM 15. The computable dimension of any computable $I$-tree with infinite height is effectively infinite.

Proof. Let $(T, I)$ be a computable $I$-tree of infinite height. There are five cases to consider.

Case 1. Let $\operatorname{ht}(T)=\omega$ and $T$ contain no infinite paths. Then $T$ contains an $\omega$-branching node $x_{0}$ with immediate successors $x_{1}, x_{2}, \ldots$ such that $\operatorname{ht}\left(T\left[x_{0}\right]\right)=\omega$, but ht $\left(T\left[x_{i}\right]\right)<\omega$ for all $i \geqslant 1$. Therefore we must have $\lim \operatorname{supht}\left(T\left[x_{i}\right]\right)=\omega$, and so Proposition 12 applies to $(T, I)$.

Case 2. Let $\operatorname{ht}(T)=\omega, T_{\text {ext }} \neq \varnothing$, and $T_{\text {ext }}$ not be finite-branching. Then there is a node $x_{0} \in T_{\text {ext }}$ with infinitely many immediate successors $x_{1}, x_{2}, \ldots$ in $T_{\text {ext }}\left(x_{0}\right.$ may also have non-extendible immediate successors). Therefore $\operatorname{ht}\left(T\left[x_{i}\right]\right)=\omega$ for all $i \geqslant 1$. Thus $\operatorname{ht}(T[y])=\omega$ for infinitely many immediate successors $y$ of $x_{0}$ in $T$, and so Proposition 12 applies to $x_{0}$.

Case 3. Let $\operatorname{ht}(T)=\omega, T_{\text {ext }} \neq \varnothing, T_{\text {ext }}$ be finite-branching, but $T$ contain a node $x$ such that the side tree $S[x]$ has height $\omega$. Obviously, $S[x]$ contains no infinite paths. As in Case 1, we conclude that $S[x]$ contains an $\omega$-branching node $x_{0}$ with immediate successors $x_{1}, x_{2}, \ldots$ in $S[x]$ such that ht $\left(S\left[x_{0}\right]\right)=\omega$, but $\operatorname{ht}\left(S\left[x_{i}\right]\right)<\omega$ for all $i \geqslant 1$.

It follows that $\left\{x_{1}, x_{2}, \ldots\right\}$ is exactly the set of all non-extendible immediate successors of $x_{0}$ in $T$, and

$$
\limsup _{i} \operatorname{ht}\left(T\left[x_{i}\right]\right)=\limsup _{i} \operatorname{ht}\left(S\left[x_{i}\right]\right)=\omega .
$$

On the other hand, $x_{0}$ may have only finitely many extendible immediate successors in $T$, since $T_{\text {ext }}$ is finitely branching. Hence Proposition 12 applies to $x_{0}$.

Case 4. Let ht $(T)=\omega, T_{\text {ext }} \neq \varnothing, T_{\text {ext }}$ be finite-branching, and all side trees in $T$ have finite height. Then we apply Proposition 13.

Case 5. Let $h t(T)>\omega$. Then Proposition 14 covers this case.

COROLLARY 16. The computable dimension of any computable $I$-tree with infinite height is $\omega$.

COROLLARY 17. No computable $I$-tree of infinite height is computably categorical.

\section{THE CASE OF SEVERAL DISTINGUISHED SUBTREES}

In conclusion, we show that all results of the present paper can be naturally generalized to the case of trees with several distinguished initial subtrees.

First, notice that while considering trees in a language with partial order $\prec$ and with $r+1$ distinguished initial subtrees $I^{0}, \ldots, I^{r}$, it is sufficient to study the case where these subtrees form the chain $I^{0} \supseteq I^{1} \supseteq$ $\ldots \supseteq I^{r}$. This follows from the well-known fact that linear basis for a finite Boolean algebra can be expressed by its generators via Boolean operations $\vee$ and $\wedge$ (see [9]).

Second, it is easy to see that in order to generalize Propositions 12-14 to the case of several subtrees we need only modify Lemmas 10, 11, and 7 correspondingly, which in turn are corollaries to Lemma 3.

Thus we need only generalize Lemma 3 to the case of several nested initial subtrees.

For $r \in \omega$, define the class $\mathbb{T}^{[r]}$ of multiple I-trees with labels from $\omega$ as follows:

$\mathbb{T}^{[r]}=\left\{(T, l) \mid T\right.$ is a finite tree with $r+1$ distinguished initial subtrees $\left.I^{0} \supseteq I^{1} \supseteq \ldots \supseteq I^{r}, l: T \rightarrow \omega\right\}$. We will write $\left(T_{1}, l_{1}\right) \leqslant\left(T_{2}, l_{2}\right)$ iff there exists an isomorphic embedding $f:\left(T_{1}, I_{1}^{0}, \ldots, I_{1}^{r}\right) \rightarrow$ $\left(T_{2}, I_{2}^{0}, \ldots, I_{2}^{r}\right)$ such that $l_{1}(x) \leqslant l_{2}(f(x))$ for all $x \in T_{1}$. Clearly, $\mathbb{T}^{[r]}$ is quasiordered by this relation. 
LEMMA 18. Let $\left\{\left(T_{i}, I_{i}^{0}, \ldots, I_{i}^{r}\right) \mid i \in \omega\right\}$ be an infinite collection of multiple $I$-trees, each with a labelling $l_{i}: T_{i} \rightarrow \omega$. Then there exist $i<j$ in $\omega$ and an embedding $f:\left(T_{i}, I_{i}^{0}, \ldots, I_{i}^{r}\right) \rightarrow\left(T_{j}, I_{j}^{0}, \ldots, I_{j}^{r}\right)$ such that $l_{i}(x) \leqslant l_{j}(f(x))$ for every $x \in T_{i}$.

Proof. For $r=0$, the statement is established in Lemma 3. We proceed by induction on $r$. As in Lemma 3, we may assume that every subtree of the form $I_{i}^{r}$ is non-empty. For each $i \in \omega$, the labelling function

$$
m_{i}: I_{i}^{r} \rightarrow \mathbb{T}^{[r-1]} \times \omega
$$

on the tree $I_{i}^{r}$ is defined as follows: for any $x \in I_{i}^{r}$, put $m_{i}(x)=\left(m_{i}^{1}(x), m_{i}^{2}(x)\right)$, where

(1) $m_{i}^{1}(x)=\left(S_{i}(x), l_{i}\left\lceil S_{i}(x)\right) \in \mathbb{T}^{[r-1]}\right.$ with finite tree

$$
S_{i}(x)=\{x\} \cup\left\{y \in T_{i} \mid y \succ x \& \forall z \preccurlyeq y\left(x \prec z \rightarrow z \notin I_{i}^{r}\right)\right\},
$$

and a labelling function $l_{i} \uparrow S_{i}(x): S_{i}(x) \rightarrow \omega$.

(2) $m_{i}^{2}(x)=l_{i}(x)$.

By Kruskal's theorem, in view of the inductive assumption, we conclude that $\mathbb{T}\left(\mathbb{T}^{[r-1]} \times \omega\right)$ is a wqo. Thus, for the collection $\left\{\left(I_{i}^{r}, m_{i}\right) \mid i \in \omega\right\}$ of elements of $\mathbb{T}\left(\mathbb{T}^{[r-1]} \times \omega\right)$, there are $i$ and $j$ for which $i<j$ and $\left(I_{i}^{r}, m_{i}\right) \leqslant\left(I_{j}^{r}, m_{j}\right)$, that is, there exists an embedding $g: I_{i}^{r} \rightarrow I_{j}^{r}$ such that $m_{i}(x) \leqslant m_{j}(g(x))$ for every $x \in I_{i}^{r}$. It follows that $l_{i}(x) \leqslant l_{j}(g(x))$ for all $x \in I_{i}^{r}$, and there exists an embedding $h_{x}: S_{i}(x) \rightarrow S_{j}(g(x))$, which respects the initial subtrees $I^{0}, \ldots, I^{r-1}$ and is such that $l_{i}(y) \leqslant l_{j}\left(h_{x}(y)\right)$ for every $y \in S_{i}(x)$.

We define a mapping $f: T_{i} \rightarrow T_{j}$ as follows:

$$
f(y)= \begin{cases}g(y) & \text { if } y \in I_{i}^{r} ; \\ h_{x}(y) & \text { if } y \notin I_{i}^{r} \text { and } y \in S_{i}(x) \text { for some } x \in I_{i}^{r} .\end{cases}
$$

As in Lemma 3, we conclude that $f$ is well defined. Now it is easy to see that $f:\left(T_{i}, I_{i}^{0}, \ldots, I_{i}^{r}\right) \rightarrow$ $\left(T_{j}, I_{j}^{0}, \ldots, I_{j}^{r}\right)$ is the desired embedding.

\section{REFERENCES}

1. R. G. Miller, "The computable dimension of trees of infinite height," forthcoming.

2. S. Lempp, C. McCoy, R. G. Miller, and R. Solomon, "Computable categoricity of trees of finite height," forthcoming.

3. S. S. Goncharov and Yu. L. Ershov, Constructive Models, Siberian School of Algebra and Logic [in Russian], Nauch. Kniga, Novosibirsk (1999).

4. Handbook of Recursive Mathematics, Vols. 1/2, Y. L. Ershov, S. S. Goncharov, A. Nerode, and J. B. Remmel (eds.), Elsevier, Amsterdam (1998).

5. S. S. Goncharov and V. D. Dzgoev, "Autostability of models," Algebra Logika, 19, No. 1, 45-58 (1980).

6. P. E. Alaev, "Autostable I-algebras," Algebra Logika, 43, No. 5, 511-550 (2004).

7. J. B. Kruskal, "Well-quasi-ordering, the tree theorem, and Vázsonyi's conjecture," Trans. Am. Math. Soc., 95, No. 2, 210-225 (1960).

8. S. G. Simpson, "Nonprovability of certain combinatorical properties of finite trees," in Harvey Friedman's Research on the Foundations of Mathematics, Stud. Log. Found. Math., Vol. 117, NorthHolland, Amsterdam (1985), pp. 87-117.

9. S. S. Goncharov, Countable Boolean Algebras and Decidability, Siberian School of Algebra and Logic [in Russian], Nauch. Kniga, Novosibirsk (1996). 